\title{
Informed Consent for Emergency Obstetric Care During COVID-19 Pandemic
}

\author{
Saswati Tripathy ${ }^{1} \cdot$ Satyajit Mohapatra ${ }^{2}$
}

Received: 14 May 2020 / Accepted: 15 June 2020 / Published online: 3 July 2020

(c) Federation of Obstetric \& Gynecological Societies of India 2020

\begin{abstract}
Informed consent process has become a challenging issue before surgery for any emergency obstetric care during this COVID pandemic. There is an increased risk of morbidity if there is a need of intensive care unit postoperatively and a risk of high mortality if patient has symptoms of COVID-19. Admission to intensive care unit adds on to the financial burden to the patient. Also, there is an increased risk of perinatal anxiety and depression during the COVID pandemic. When an asymptomatic carrier develops symptoms of COVID after delivery or caesarean section, the morbidity increases. So we have designed an informed consent form for patients undergoing emergency obstetric surgeries incorporating some points specific for COVID-19.
\end{abstract}

Keywords COVID-19 $\cdot$ Pandemic $\cdot$ Informed consent

\section{Introduction}

Pregnancy and delivery pose unique challenges during the coronavirus disease (COVID-19) pandemic due to altered maternal immune system [1]. In India, the institutional deliveries have been increased in last decade and there is a huge burden in hospital settings during this pandemic. Obtaining a valid informed consent in humanitarian emergencies such as COVID-19 is a challenge. There are practical difficulties in identifying a COVID-19 positive patient. According to the recent ICMR (Indian Council of Medical Research) guidelines, if a woman meets the criteria for COVID-19 testing like travel history from an affected area or close contact

Saswati Tripathy is a Professor of Obstetric and Gynaecology, SRM Medical College Hospital and Research Centre,

Kattankulathur, Tamil Nadu, India. Satyajit Mohapatra is a Professor of Pharmacology, SRM Medical College Hospital and Research Centre, Kattankulathur, Tamil Nadu, India.

Saswati Tripathy

drsaswati78@gmail.com

1 Department of Obstetric and Gynaecology, SRM Medical College Hospital and Research Centre, Kattankulathur, Tamil Nadu, India

2 Department of Pharmacology, SRM Medical College Hospital and Research Centre, Kattankulathur, Tamil Nadu, India with a confirmed case of COVID-19, she should be tested. Until RT-PCR (real-time reverse transcription polymerase chain reaction) test results are available, she should be treated as though she has confirmed COVID-19 [2]. So during that time it is a challenge to obtain the consent from the patient covering all aspects of the informed consent process. Informed consent is a continuous process involving three main components-providing relevant information, ensuring competence, ensuring comprehension and voluntariness [3]. In addition, the decisional capacity of the hospitalized patient would be very low during this pandemic. Also, there is an increased risk of the need of intensive care unit (ICU) for ventilator facilities as well as an associated risk of high morbidity and mortality if the patient exhibits symptoms of COVID-19 in the postoperative period.

Therefore, we have devised a new informed consent format for all patients undergoing emergency obstetric surgeries incorporating a few points specific for the COVID-19 disease.

\section{Discussion}

Informed consent for emergency obstetric care, which is a critical component of surgical practice, has become a challenging issue in the time of COVID-19 infection. There are few specific requirements which need to be discussed pre-op 
during this pandemic with the patient and the relatives, in order to avoid any unnecessary medico legal issues. Reports have shown that over a recent 5 -year period, $65 \%$ of medico legal cases involving informed consent were surgical and only $21 \%$ of these cases were decided in favor of the surgeon [4]. It is mandatory that prior to obtaining consent for the proposed surgery, the obstetrician must provide the patient with detailed information about the nature of the surgery, the expected risk and benefits, alternate treatments and the consequence of not having the surgery [5].

In India, the need for ICU and ventilator support in the post-op period, if required, must be discussed with the patient's relative as it involves an increased financial burden. There are also concerns about the lack of pre-op COVID testing due to paucity of commercial testing kits. But preop COVID testing should be done for elective surgeries if the facilities are available. A normal patient may develop or contract coronavirus infection in the peri- or postoperative period which should be again documented in the consent form. All the potential risks, short term or long term should be informed to the patient.

Hence, a detailed modified consent form was designed exclusively for the patients who require emergency obstetric surgeries during this pandemic.

\section{Compliance with Ethical Standards}

Conflict of interest The author declares thatthere is no conflict of interest.

Ethical Statement This article does not contain any studies with animals performed by any of the authors. 


\section{Appendix}

\section{INFORMED CONSENT DURING COVID-19}

(Should be translated in local language)

Facility: Covid / Non Covid ward

COVID Status: Positive/ Negative/ Unknown

Place of Consent: Triage area/ at admission/ before surgery

Contact history with confirmed case of COVID: Positive/ Negative

Travel history to affected area: suggestive/non-suggestive

History of Fever/ Cough/ Respiratory symptoms: Yes/ No

Resident of containment zone: Yes/No

I

(patient name) Age

Address having Hospital No/

Aaadhar no understand that I am admitted for an emergency/elective surgery during the

COVID-19 pandemic.

I understand that COVID-19 is extremely contagious and is believed to spread by person-to-person contact.

However, given the nature of the virus, I understand that there is an inherent risk of becoming infected with COVID19 by virtue of proceeding with this emergency/elective surgery.

I hereby acknowledge and assume the risk of becoming infected with COVID-19 through this surgery, and I give my express permission for the treating doctor Dr and concerned staffs to proceed with the same.

I understand that, even if I have been tested for COVID and received a negative test result, the tests in some cases may fail to detect the virus or I may have contracted COVID after the test. I understand that, if I have a COVID-19 infection, and even if I do not have any symptoms for the same, proceeding with this surgery can lead to a higher chance of complication and death.

I understand that possible exposure to COVID-19 before/during/after my surgery may result in the following: a positive COVID-19 diagnosis, extended quarantine/self-isolation, additional tests, hospitalization that may require medical therapy, Intensive care treatment, and possible need for intubation/ventilator support, short-term or longterm intubation, other potential complications, and the risk of death.

I understand that COVID-19 may cause additional risks, some or many of which may not currently be known at this time, in addition to the risks described herein, as well as those risks for the treatment/procedure/surgery itself.

I have been counselled adequately by Dr and staffs in the language that $\mathrm{I} / \mathrm{my}$ patient can understand.

I have been given the option to refuse /defer my surgery. However, I understand all the potential risks, including but not limited to the potential short-term and long-term complications related to COVID-19, and I would like to proceed with my desired surgery. I will not hold the doctor, the hospital or its staffs morally or legally accountable for any reason.

This is to state that I have comprehensively explained the information above along with the explanation provided by the doctor to the patient and/or her attendant have informed me that they have understood the information completely.

Signature of Patient

Name of the patient:

Address:

Contact No:

Date and Time:

Signature of Surgeon:

Name \& Designation:

Address:

Contact no:

Date and Time
Signature of Legally acceptable authorizer (LAR)/Witness

Name of the LAR/Witness 


\section{References}

1. Putra M, Kesavan MM, Brackney K, Hackney DN, Roosa MKM. Forecasting the impact of coronavirus disease during delivery hospitalization: an aid for resources utilization. Am J Obstet Gynecol MFM. 2020. https://doi.org/10.1016/j.ajogmf.2020.100127.

2. Guidance for Management of Pregnant Women in COVID-19 Pandemic. ICMR- National Institute for Research in Reproductive Health. https://www.icmr.gov.in/pdf/covid/techdoc/Guidance_ for_Management_of_Pregnant_Women_in_COVID19_Pande mic_12042020.pdf.

3. National guidelines for ethics committee reviewing biomedical and health research during COVID-19 pandemic. Indian Council of Medical Research. April 2020.

4. Canadian Medical Protective Association. Risk Fact Sheet CMPA. www.cmpa-acpm.ca/documents/10179/300031190/informed_ consent-e.pdf. Accessed 1 Mar 2016.

5. Bhattacharya $\mathrm{N}$, Bhattacharya $\mathrm{K}$. Informed consent for surgery during COVID-19. Indian J Surg. 2020. https://doi.org/10.1007/ s12262-020-02283-y.

Publisher's Note Springer Nature remains neutral with regard to jurisdictional claims in published maps and institutional affiliations.
About the Author

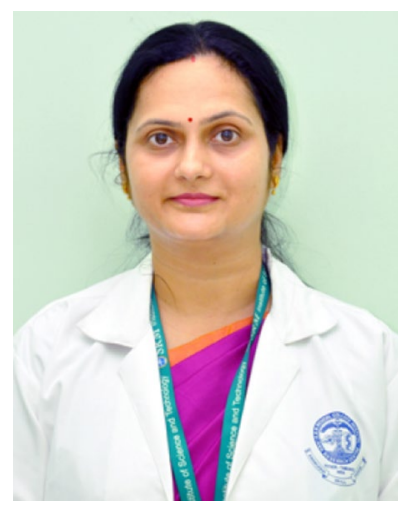

Dr. Saswati Tripathy is currently working as Professor of Obstetrics and Gynaecology at SRM Medical College Hospital and Research Centre, Tamil Nadu. She has done her post-graduation from the prestigious SCB Medical College, Cuttack, Odisha. She is a fellow of Indian College of Obstetricians and Gynecologists. Her area of interests are operative obstetric care, high risks pregnancy and infertility. 